\title{
Trombectomía más fleboextracción: una técnica alternativa en el manejo de la enfermedad hemorroidal externa trombosada
}

\section{Thrombectomy combined with vein stripping: An alternative technique to treat thrombosed external hemorrhoid disease}

Carlos Edmundo Martínez-Jaramillo, ${ }^{*}$ @D Nairo Javier Senejoa-Núñez, ${ }^{1}$ Heinz Orlando Ibáñez-Varela,, Luis Francisco Miranda-Ruiz, ${ }^{1}$ Roberto José Rodríguez-Flórez, ${ }^{2}$ Nicolás Betancur, ${ }^{2}$ Laura Daniela Ramírez-Melo, ${ }^{3}$ María Camila Rubio-Rubiano. ${ }^{3}$

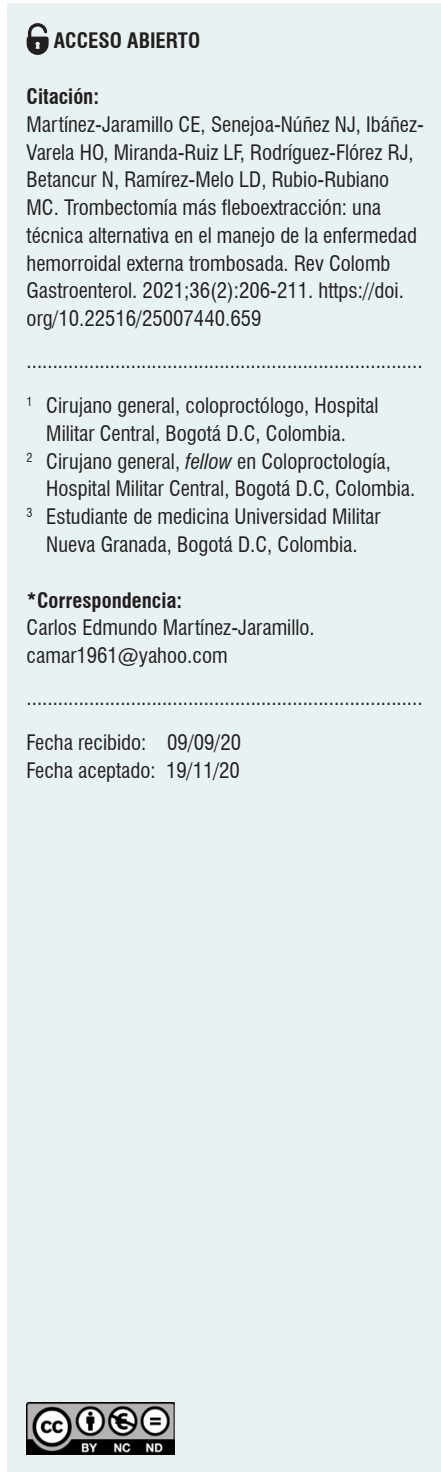

\section{Resumen}

Objetivo: evaluar los resultados de la trombectomía más fleboextracción como alternativa en el manejo de la enfermedad hemorroidal externa trombosada. Materiales: estudio observacional, cuantitativo, retrospectivo, transversal y descriptivo de los pacientes que consultaron y se sometieron a la trombectomía más fleboextracción como técnica alternativa en el manejo de la enfermedad hemorroidal externa trombosada en el servicio de coloproctología del Hospital Militar Central desde el primero de enero de 2014 hasta el 31 de diciembre de 2016. Resultados: se encontró que 197 pacientes consultaron por hemorroides externas trombosadas, el $71 \%$ eran hombres y el promedio de edad para el grupo de manejo conservador fue de 48,5 años, frente al promedio del grupo de manejo quirúrgico, que fue de 43,2 años. El seguimiento promedio posterior al inicio del tratamiento médico o quirúrgico fue de 16 meses. En el grupo de manejo quirúrgico, la tasa de recurrencia fue del 7,2 \% y el intervalo de recurrencia fue 29,2 meses. Conclusiones: los pacientes a quienes se les practicó la técnica de trombectomía más fleboextracción presentaron una menor tasa de retrombosis hemorroidal, menor tasa de dolor y sangrado posoperatorio. La trombectomía hemorroidal con fleboextracción es una técnica bien tolerada en el consultorio, que se puede realizar sin anestesia, a diferencia del estándar de manejo actual con hemorroidectomía externa en elipse, que requiere anestesia local, regional o general, y una sala de procedimientos quirúrgicos que deja un defecto mayor en la piel por el tejido resecado, un mayor tiempo de cicatrización y de dolor posoperatorio.

\section{Palabras clave}

Hemorroides, trombectomía, recurrencia, tratamiento, resultado.

\begin{abstract}
Objective: To evaluate the results of thrombectomy combined with vein stripping as an alternative to treat external thrombosed hemorrhoid disease. Materials and methods: This is an observational, quantitative, retrospective, cross-sectional and descriptive study of the patients who underwent thrombectomy combined with vein stripping as an alternative technique to treat thrombosed external hemorrhoid disease at the Coloproctology Service of the Hospital Militar Central from January 1, 2014, to December 31, 2016. Results: It was found that 197 patients consulted due to thrombosed external hemorrhoids. $71 \%$ were men. The average age for the conservative treatment group vs. the surgical treatment group was 48.5 vs. 43.2 years, respectively. The average follow-up time after initiation of medical or surgical treatment was 16 months. In the surgical treatment group, the recurrence rate was $7.2 \%$ and the recurrence interval was 29.2 months. Conclusions: Patients who underwent thrombectomy combined with vein stripping had a lower recurrence rate of thrombosed hemorrhoids, a lower pain score, and less postoperative bleeding. Hemorrhoid thrombectomy combined with vein stripping is a well-tolerated technique that can be performed at the doctor's office without anesthesia, unlike the current management standard with external elliptical hemorrhoidectomy, which that requires local, regional, or general anesthesia, and a surgical room, leaving a larger skin defect following the resection of the tissue, with longer healing time and greater postoperative pain.
\end{abstract}

\section{Keywords}

Hemorrhoids; Thrombectomy; Recurrence; Treatment; Outcome. 


\section{INTRODUCCIÓN}

Las almohadillas o cojinetes hemorroidales son estructuras compuestas de tejido mucoso y submucoso, plexos venosos hemorroidales, tejido conectivo elástico y algunas fibras de músculo liso (corrugador del ano como parte terminal de las fibras musculares longitudinales del recto) que se insertan en la piel (1-3). Entre sus funciones se encuentran ayudar a la continencia fina y al cierre hermético del canal anal en reposo, y proteger el esfínter durante la defecación (1, $4,5)$. Aunque son estructuras anatómicamente normales, el término enfermedad hemorroidal (EH) hace referencia al proceso patológico sintomático (6).

La enfermedad hemorroidal es una condición clínica muy prevalente en la población adulta y es un frecuente motivo de consulta en el servicio de urgencias. Se estima que aproximadamente el $5 \%$ de la población adulta sufre de enfermedad hemorroidal, y es mucho más frecuente en mayores de 50 años, alcanzando una frecuencia de hasta el $50 \%(7,8)$. En Colombia no hay estudios de registro epidemiológicos de esta enfermedad.

La etiología de la EH aún no es muy clara; sin embargo, se han propuesto 4 teorías. La teoría más aceptada es la mecánica, en la que el esfuerzo defecatorio prolongado (mal hábito defecatorio) y la constipación causan arrastre, estiramiento y daño de las fibras de colágeno de los tejidos que recubren el canal anal (9). Como segunda teoría, señala la compresión venosa que obstruye el retorno sanguíneo de los plexos hemorroidales causado por el útero durante el embarazo, momento en el que con frecuencia las hemorroides se tornan sintomáticas. La tercera teoría explicaría el origen de la trombosis hemorroidal externa, con la vasodilatación presentada con el consumo de algunos alimentos (grasas, alcohol, condimentos), actividades que aumentan la presión intraabdominal (como tos, esfuerzo al vomitar, sentadillas o postura acurrucada por períodos largos) o calor local (sentarse en superficies calientes); estos eventos muy frecuentemente son referidos por el paciente con la trombosis hemorroidal externa; la vasodilatación y el aumento de presión intravenosa hemorroidal causan elongación y despulimiento del endotelio, exposición de tejido conectivo subendotelial ocasionando agregación plaquetaria y trombosis hemorroidal. La cuarta y última teoría es la vascular, que consiste en la presencia de fistulas arteriovenosas y ausencia de válvulas en los paquetes hemorroidales, que causan un aumento del volumen sanguíneo y elongación de los paquetes hemorroidales y el recubrimiento anal, y es el soporte para la realización de procedimientos como la ligadura arterial selectiva hemorroidal (10). Muchos de los pacientes con trombosis hemorroidal externa refieren este como primer evento y no tienen ningún antecedente $o$ síntomas de prolapso hemorroidal interno.
Las hemorroides se clasifican en internas y externas y dependen de su relación con la línea dentada $(5,6)$. Las hemorroides internas se ubican proximales a esta, se encuentran recubiertas por epitelio columnar de origen endodérmico y son inervadas por fibras viscerales que no transmiten dolor. La enfermedad hemorroidal interna se clasifica en 4 grados con base en el nivel de prolapso hemorroidal con respecto al borde anal y se utiliza para seleccionar y evaluar los tratamientos (11).

Las hemorroides externas tienen una ubicación distal a la línea dentada, están cubiertas por epitelio escamoso que deriva del ectodermo y su inervación es somática, por lo que tienden a ser dolorosas cuando se presentan trombosis $(6,12)$. La trombosis de los paquetes hemorroidales externos es la principal complicación de la enfermedad hemorroidal externa (EHE).

La presentación clínica de la trombosis hemorroidal externa está dada por dolor anal intenso, continuo y de inicio súbito, acompañado de sensación de un bulto o masa en la región anal y, en algunos casos, unos días más tarde (48 a 72 horas) puede presentarse sangrado anal cuando se acompaña de necrosis de la pared del paquete hemorroidal trombosado con la consecuente evacuación parcial del trombo.

El tratamiento estándar de esta complicación en las primeras 72 horas se basa en el manejo médico sintomático $y$, en los casos más graves, el tratamiento es quirúrgico. Este último consiste en la resección elíptica del paquete hemorroidal externo trombosado, bajo anestesia locorregional o general. Las principales indicaciones y objetivos del manejo quirúrgico se presentan en la Tabla $\mathbf{1}$.

Tabla 1. Indicaciones y objetivos del manejo quirúrgico en enfermedad hemorroidal externa trombosada

\section{Indicaciones:}

Hemorroide externa trombosada $>2 \mathrm{~cm}$

Dolor grave que no mejora con analgésicos

Masa anal de gran tamaño que persiste después de 3 semanas

Necrosis de la pared o sangrado de la EHE trombosada

Deseo del paciente para que se le solucione la sensación de masa

Episodios a repetición (más de 3 episodios de trombosis en 1 año, independientemente del tamaño del paquete)

Objetivos:

Mejorar la masa anal

Mejorar el dolor anal

Evitar la retrombosis

Retornar pronto a las actividades diarias

Lograr un tratamiento de bajo costo y fácil resolución en el consultorio 
Los efectos en este tipo de cirugía son el dolor de la herida y un defecto de piel de tamaño variable producido por la elipse de piel resecada, que puede tardar en cicatrizar entre 2 y 4 semanas, así como la disponibilidad de una sala de procedimientos quirúrgicos, que implica mayor costo y tiempo para solucionar este problema.

Con la trombectomía simple se han reportado tasas de retrombosis hasta del $33 \%$ medidas a 24 meses (13-16). La trombectomía con incisión amplia en la piel con fleboextracción reduciría a menos del $3 \%$ de la retrombosis, reduciría el dolor posoperatorio, acortaría el tiempo de cicatrización, generaría un pronto retorno a las actividades diarias y el manejo se hace de una manera ágil y a bajo costo.

En la EHE trombosada que no cumpla los criterios quirúrgicos se indica el manejo médico. Este puede ir desde la utilización de compresas frías en los primeros 3 días hasta la realización de baños de asiento con agua tibia (entre 38-40 ${ }^{\circ} \mathrm{C}$ ) en los siguientes 15 días, en los que se puede incluir agentes antiinflamatorios y astringentes de piel como caléndula o sulfato de magnesio. También se recomienda ablandadores de materia fecal, evitar malos hábitos defecatorios, suspender alimentos vasodilatadores o irritantes de la piel perianal y el uso de flebotónicos y analgésicos orales.

\section{MATERIALES Y MÉTODOS}

Se realizó una búsqueda en la base de datos de historias clínicas electrónicas de la institución. Se identificaron los pacientes diagnosticados y tratados con enfermedad hemorroidal externa trombosada desde el 1 de enero de 2014 hasta el 31 de diciembre de 2016 en el servicio de coloproctología del Hospital Militar Central. Se obtuvieron historias clínicas completas de 197 pacientes con diagnóstico de primer episodio de EHE trombosada. Se describieron las características demográficas de la población, el tipo de manejo (quirúrgico y médico); desenlaces como dolor, sangrado, infección (complicaciones tempranas) hasta 30 días luego de iniciado el tratamiento.

Se realizó una revisión de las historias con un seguimiento mínimo de 12 meses posteriores al tratamiento inicial con el fin de evaluar la tasa de retrombosis, tiempo de cicatrización y complicaciones tardías (hasta 1 año luego del inicio del tratamiento) como sangrado, infección y dolor crónico.

El manejo quirúrgico estándar en el servicio de coloproctología del Hospital Militar Central para el manejo de la enfermedad hemorroidal externa trombosada fue la técnica de trombectomía con incisión amplia de piel más fleboextracción, que consta de los siguientes pasos (Figura 1).

1. Alistamiento: además del consentimiento informado, se debe explicar el procedimiento al paciente, sus objetivos y beneficios. Cuando el paciente esté motivado y tranquilo se inicia el procedimiento. Debe tenerse disponible los siguientes materiales: hoja de bisturí número 15, pinza Kelly, gasas precortadas y guantes estériles o de manejo.

2. Exposición de la región anal: se ubica al paciente en posición de navaja (jackknife), se realiza la separación de los glúteos con cintas adhesivas (esparadrapo de tela) o mediante la separación manual de glúteos por un ayudante en posición de Sims.

3. El paquete hemorroidal externo trombosado se tracciona digitalmente logrando una eversión y exposición en toda su circunferencia para facilitar su fijación y abordaje.

4. Previa asepsia y antisepsia, sin anestesia, con un bisturí número 15 y su filo ubicado hacia arriba (para disminuir el dolor), se incide sobre el área de mayor necrosis destechando en sentido longitudinal y radiado (teniendo como centro el ano) en un $90 \%$ de la superficie del paquete hemorroidal. Se debe realizar una incisión por cada paquete hemorroidal externo trombosado.

5. Se realiza extracción del coágulo con presión digital.

6. Con una pinza Kelly se hace el pinzamiento y la extracción por tracción selectiva del plexo vascular hemorroidal, similar a la fleboextracción realizada en el tratamiento de la insuficiencia venosa de miembros inferiores.

7. Se verifica la hemostasia y colocación de tapón de gasa anal.

\section{RESULTADOS}

Se evaluó un total de 197 historias de pacientes con diagnósticos de enfermedad hemorroidal externa trombosada, de los cuales 142 ( $72 \%$ ) fueron sometidos a manejo médico y 55 pacientes $(27,9 \%)$ fueron tratados quirúrgicamente de acuerdo con las indicaciones propuestas en la Tabla $\mathbf{1}$. No se encontraron diferencias significativas entre el sexo y la edad. El seguimiento promedio posterior al inicio del tratamiento médico o quirúrgico fue de 16 meses. La tasa de recurrencia fue del 7,2\% y el intervalo de recurrencia fue de 29,2 meses en el grupo quirúrgico. No se evidenciaron complicaciones infecciosas durante el tiempo de seguimiento en los 2 grupos de pacientes (Tabla 2).

\section{DISCUSIÓN}

La enfermedad hemorroidal externa trombosada es una condición quirúrgica común. A pesar de que se ha practicado una variedad de estrategias de tratamiento, la falta de datos comparativos prospectivos sobre el tratamiento y los resultados a largo plazo es sorprendente; se han encontrado como posibles explicaciones que su manejo se considera sencillo con un resultado predecible, la falta de innovación en nuevas técnicas de manejo que lo hacen poco atractivo 

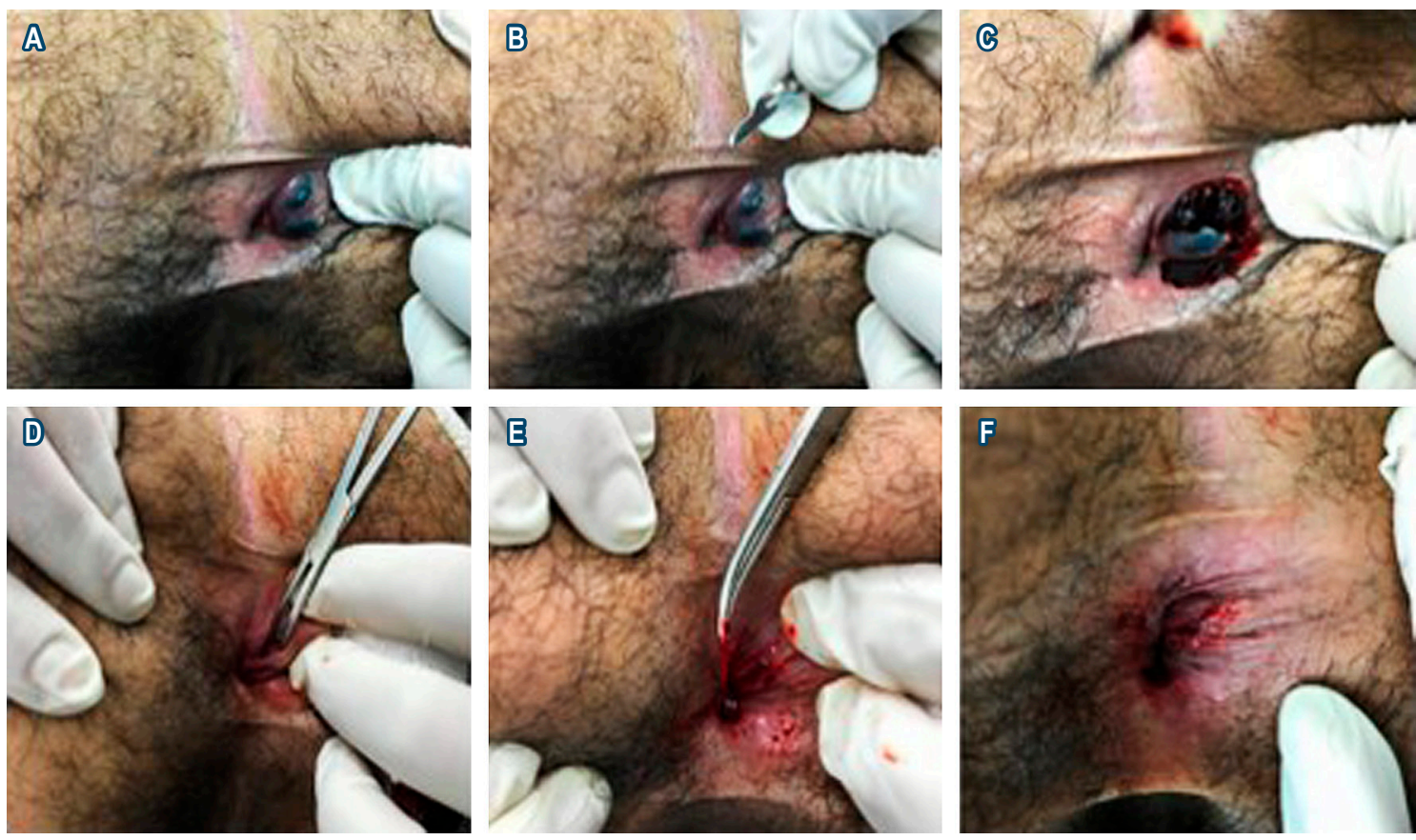

Figura 1. Técnica de trombectomía más fleboextracción. A. Fijación y eversión digital de 2 paquetes hemorroidales externos trombosados. B. Bisturí número 15 y su filo hacia arriba. C. Una incisión longitudinal radiada del $90 \%$ de la superficie de cada uno de los paquetes hemorroidales trombosados, compresión y extracción de los coágulos. D. Búsqueda, pinzamiento suave y cuidadoso de los vasos hemorroidales externos. E. Fleboextracción de los vasos hemorroidales externos por tracción con la pinza Kelly. F. Revisión del área de la trombectomía y fleboextracción, presión local en caso de sangrado. Se deja una gasa en esta región al finalizar el procedimiento, la cual debe mantenerse por 6 horas antes de ser removida. El paciente puede utilizar una gasa en la región anal por 2 o 3 días más, la cual se debe cambiar 2 o 3 veces al día. Se receta acetaminofén oral cada 8 horas por 2 días.

Tabla 2. Resultados, recurrencia y complicaciones del tratamiento hemorroidal

\begin{tabular}{|lcc|}
\hline \multicolumn{1}{|c}{ Factor } & $\begin{array}{c}\text { Grupo de manejo } \\
\text { médico }(\mathbf{n}=\mathbf{1 4 2})\end{array}$ & $\begin{array}{c}\text { Grupo de manejo } \\
\text { quirúrgico ( } \mathbf{n = 5 5}\end{array}$ \\
\hline Edad (años) & 48,5 & 43,2 \\
\hline $\begin{array}{l}\text { Sexo (\%) } \\
\text { - Hombre }\end{array}$ & 57,8 & 70,9 \\
- Mujer & 42,2 & 29,1 \\
\hline Seguimiento (meses) & 16,3 & 16,2 \\
\hline Tasa de recurrencia & 2,8 & 7,2 \\
\hline $\begin{array}{l}\text { Tiempo hasta la recurrencia } \\
\text { (meses) }\end{array}$ & 7,2 & 29,2 \\
\hline
\end{tabular}

como tema de estudio y la limitación para realizar estudios prospectivos por el volumen poblacional; además, la trombectomía para el manejo de la EHE trombosada históri- camente ha tenido mala fama y poca aceptación entre los cirujanos debido a su alta tasa de recurrencia.

Comparar los resultados del manejo médico frente al quirúrgico no es fácilmente homologable, por lo cual se ha planteado que el punto de decisión entre que método escoger debe ser el tiempo de resolución del dolor en el manejo médico y la resolución del dolor, la masa anal, el sangrado, la curación de la herida, la satisfacción del paciente, los costos y el tiempo requerido por el paciente y el cirujano para resolver el problema en los casos de manejo quirúrgico (13).

La resolución completa del dolor en los pacientes sometidos a manejo quirúrgico se logra cerca del cuarto día posterior al procedimiento, con respecto al manejo conservador, en el cual puede haber persistencia promedio hasta el día 24 (13). Nuestros datos muestran que en el $81,9 \%$ de los pacientes llevados a cirugía no hay presencia de dolor posterior a las 72 horas del procedimiento, no siendo así en el grupo de manejo conservador donde persiste el dolor hasta en un $92,2 \%$. Se plantean estrategias de manejo médico adicionales como adición de nitroglicerina o nifedipina tópica (13). 
Cavcić y colaboradores (17), Greenspon y colaboradores (13), y Perrotti y colaboradores (18) han planteado diferentes estrategias para en el manejo médico de las hemorroides externas trombosadas, principalmente usando ablandadores de heces, analgésicos locales o sistémicos combinados con nitroglicerina o nifedipina tópica y han mostrado mejores resultados que el uso solo de analgésicos.

No hay evidencia en la literatura de que el tratamiento conservador tenga mejores resultados en los casos de consulta temprana posterior al inicio de los síntomas; esta práctica parece estar basada en juicios transmitidos a lo largo de los años.

Greenspon y colaboradores (13) mostraron en un estudio retrospectivo en el cual se comparó el manejo conservador frente al quirúrgico de hemorroides externas trombosadas una recurrencia en el grupo de manejo conservador de $25,4 \%$ con tiempo promedio de recurrencia a los 7,1 meses, en contraste con el grupo de manejo quirúrgico, en el cual se presentó una recurrencia del $6,3 \%$ con un tiempo promedio de recurrencia de 25 meses. Con los datos extraídos de nuestra población con la técnica descrita se logró disminuir la tasa de recurrencia y aumentar el tiempo de la misma, inclusive considerándose que se trata de un segundo episodio independiente.

\section{CONCLUSIONES}

La trombectomía con incisión amplia de piel más fleboextracción mejora los resultados con menor tasa de recurrencia y cicatrización más rápida, por lo que resulta ser una técnica eficiente a bajo costo. Este es un procedimiento de consultorio, que resuelve de manera rápida y eficiente la sensación de masa, dolor y sangrado hemorroidal sin el requerimiento de ser llevado a un quirófano. Como ventaja adicional, tiene la facilidad de ser realizado sin la necesidad de utilización de anestesia locorregional o general, y se obtienen resultados con un menor tiempo de cicatrización y dolor perianal, una tasa de retrombosis de un $3 \%$ medido a 24 meses y una excelente satisfacción global del paciente. Es un procedimiento con una técnica fácil, reproducible y de bajo costo. Valdría la pena validar la técnica diseñando un estudio clínico controlado aleatorizado, comparando la resección en elipse del paquete hemorroidal externo trombosado con la trombectomía amplia más fleboextracción y darle consistencia a nuestros hallazgos con la experiencia de otros centros que puedan implementar la técnica y confirmar los beneficios planteados.

\section{REFERENCIAS}

1. Cintron J, Abcarian AM, Abcarian H, Makiewicz K, Brand MI. Hemorrhoids. En: Abcarian H, Cintron J, Nelson R (editores). Complications in anorectal surgery. Prevention and management. Springer; 2017. p. 61-108. https://doi.org/10.1007/978-3-319-48406-8 4

2. Sanchez C, Chinn BT. Hemorrhoids. Clin Colon Rectal Surg. 2011;24(1):5-13. https://doi.org/10.1055/s-0031-1272818

3. Thomson WH. The nature of haemorrhoids. Br J Surg. $1975 ; 62(7): 542-52$. https://doi.org/10.1002/bjs.1800620710

4. Aigner F, Gruber H, Conrad F, Eder J, Wedel T, Zelger B, et al. Revised morphology and hemodynamics of the anorectal vascular plexus: impact on the course of hemorrhoidal disease. Int J Colorectal Dis. 2009;24(1):105-13. https://doi.org/10.1007/s00384-008-0572-3

5. Schubert MC, Sridhar S, Schade RR, Wexner SD. What every gastroenterologist needs to know about common anorectal disorders. World J Gastroenterol. 2009; 15(26):3201-9. https://doi.org/10.3748/wjg.15.3201

6. Sun Z, Migaly J. Review of Hemorrhoid Disease: Presentation and Management. Clin Colon Rectal Surg. 2016;29(1):22-9.

https://doi.org/10.1055/s-0035-1568144
7. Sneider EB, Maykel JA. Diagnosis and management of symptomatic hemorrhoids. Surg Clin North Am. 2010;90(1):17-32. https://doi.org/10.1016/j.suc.2009.10.005

8. Gençosmanoğlu R, Sad O, Koç D, Inceoğlu R. Hemorrhoidectomy: open or closed technique? A prospective, randomized clinical trial. Dis Colon Rectum. 2002;45(1):70-5. https://doi.org/10.1007/s10350-004-6116-1

9. Talley NJ, Lasch KL, Baum CL. A gap in our understanding: chronic constipation and its comorbid conditions. Clin Gastroenterol Hepatol. 2009; 7(1):9-19. https://doi.org/10.1016/j.cgh.2008.07.005

10. Wronski K. Etiology of thrombosed external hemorrhoids. Postepy Hig Med Dosw (Online). 2012;66:41-4.

11. Mott T, Latimer K, Edwards C. Hemorrhoids: Diagnosis and Treatment Options. Am Fam Physician. 2018;97(3):172-179.

12. Margetis N. Pathophysiology of internal hemorrhoids. Ann Gastroenterol. 2019;32(3):264-272. https://doi.org/10.20524/aog.2019.0355

13. Greenspon J, Williams SB, Young HA, Orkin BA. Thrombosed external hemorrhoids: outcome after conservative or surgical management. Dis Colon Rectum. 2004;47(9):1493-8. https://doi.org/10.1007/s10350-004-0607-y 
14. Abramowitz L, Sobhani I, Benifla JL, Vuagnat A, Daraï E, Mignon M, et al. Anal fissure and thrombosed external hemorrhoids before and after delivery. Dis Colon Rectum. 2002;45(5):650-5. https://doi.org/10.1007/s10350-004-6262-5

15. Gebbensleben O, Hilger Y, Rohde H. Do we at all need surgery to treat thrombosed external hemorrhoids? Results of a prospective cohort study. Clin Exp Gastroenterol. 2009;2:69-74.

https://doi.org/10.2147/ceg.s5986

16. Janicke DM, Pundt MR. Anorectal disorders. Emerg Med Clin North Am. 1996;14(4):757-88.

https://doi.org/10.1016/s0733-8627(05)70278-9
17. Cavcić J, Turcić J, Martinac P, Mestrović T, Mladina R, Pezerović-Panijan R. Comparison of topically applied $0.2 \%$ glyceryl trinitrate ointment, incision and excision in the treatment of perianal thrombosis. Dig Liver Dis. 2001;33(4):335-40. https://doi.org/10.1016/s1590-8658(01)80088-8

18. Perrotti P, Antropoli C, Molino D, De Stefano G, Antropoli M. Conservative treatment of acute thrombosed external hemorrhoids with topical nifedipine. Dis Colon Rectum. 2001;44(3):405-9.

https://doi.org/10.1007/BF02234741 